\title{
Establishment of RANKL-highly sensitive RAW264 subclones in accordance with high expression of TPC2
}

\author{
Takuya Notomi", Akiko Hiyama and Tadashige Nozaki \\ Department of Pharmacology, Osaka Dental University, 8-1 Kuzuhahanazono-cho, Hirakata, Osaka 573-1121, Japan
}

\begin{abstract}
Osteoclast differentiation is one of the critical steps that control bone mass levels during bone remodeling. However, the molecules involved in osteoclastogenesis are still incompletely understood. The pre-osteoclast cell lines RAW264 and 264.7 are widely used to study osteoclast differentiation, however, their response to RANKL stimulation is relatively lower than that of primary pre-osteoclast. Here, we established novel RAW264 subclones. The subclones showed various responses to RANKL stimulation and their tartrate-resistant acid phosphatase (TRAP) activity was strongly correlated with the mRNA expression of two-pore channel subtype 2 (TPC2). TRAP activity in Clone15 and 10 was higher and lower when compared with that of RAW264.7 cells, respectively. We conclude that the subclones established in our study are useful tools to study osteoclast biology. Moreover, we demonstrated that TPC2 expression levels influence osteoclastogenesis in RAW264 subclones.
\end{abstract}

\section{Introduction}

Osteoclasts are multinucleated cells that resorb bone matrix. The mechanism of osteoclastogenesis has been extensively investigated [1-4]. These studies have shown that the receptor activator of nuclear factor- $\kappa \mathrm{B}$ (RANK)/RANK ligand (RANKL) pathway induces changes in intracellular $\mathrm{Ca}^{2+}\left(\left[\mathrm{Ca}^{2+}\right]_{\mathrm{i}}\right)$ levels. This leads to $\mathrm{Ca}^{2+} /$ calcineurindependent dephosphorylation and activation of nuclear factor of activated T cells 1 (NFATc1), which then translocates from the cytosol to the nucleus $[3,4]$. Moreover, in regard to osteoclastic molecules associated with the $\left[\mathrm{Ca}^{2+}\right]_{\mathrm{i}}$ pathway, we have previously reported that a lysosomal $\mathrm{Ca}^{2+}$ channel, namely the two-pore channel subtype 2 (TPC2), plays a role in osteoclastogenesis [4,5].

TPC2 is a member of the $\mathrm{Ca}^{2+}$-permeable channels, which are activated by nicotinic acid adenine dinucleotide phosphate (NAADP), a potent $\mathrm{Ca}^{2+}$-mobilizing cellular messenger [6,7]. TPC2 has been identified as a lysosomal $\mathrm{Ca}^{2+}$ channel and thus it is not localized in the endosome, endoplasmic reticulum (ER), Golgi apparatus or mitochondria. NAADP evokes $\mathrm{Ca}^{2+}$ release from the lysosome via TPC2 [6], which leads to further $\mathrm{Ca}^{2+}$ release from the ER via inositol triphosphate receptors or ryanodine receptors [6,7]. Notably, we have identified TPC2 as a previously unknown regulator of osteoclastogenesis. Previously, we showed by TPC2 gene knockdown in RAW267.4 that TPC2 promotes osteoclast differentiation by regulating $\left[\mathrm{Ca}^{2+}\right]_{\mathrm{i}}$ and NFATc1 localization [4] in the RANK/RANKL pathway.

RAW264 is a murine macrophage cell line which can differentiate into osteoclast-like cells in response to RANKL stimulation. Thus, these cells are widely used to investigate the mechanism of osteoclastogenesis [8-10]. Although many researchers have used RAW264 cells to investigate osteoclastogenesis, the response of RAW264 to RANKL stimulation is low when compared to that of primary pre-osteoclasts. As such, RAW264 subclones, such as RAW264.7, were generated by some research groups [8,9]. This led us to investigate whether RAW264 subclones respond differently to RANKL stimulation and whether this response depends on TPC2 expression levels. Here, we generated RAW264 subclones with highly efficient osteoclastogenesis in response to RANKL. Moreover, we demonstrated that TPC2 expression levels modulate osteoclastogenesis in RAW264 subclones.

\section{Material and methods}

\section{Cell culture and establishment of RAW264 subclones}

RAW 264 and 264.7 mouse osteoclast precursor-like cells (RIKEN, Saitama, Japan, and American Type Culture Collection, Manassas, VA, USA) were cultured in DMEM medium (GE Healthcare Japan, Tokyo, Japan) supplemented with $10 \%$ FBS and $1 \%$ penicillin and streptomycin. All cells were maintained at $37{ }^{\circ} \mathrm{C}$ and $5 \% \mathrm{CO}_{2}$ in a humidified atmosphere. All chemicals used were purchased from Wako Pure Chemical Industries (Osaka, Japan) unless otherwise specified.

For osteoclast differentiation, cells were seeded at $2.0 \times 10^{5} \mathrm{cells} / \mathrm{cm}^{2}$ in the presence of soluble RANKL (sRANKL, $50 \mathrm{ng} / \mathrm{mL}$; Oriental Yeast, Tokyo, Japan). TRAP staining was performed as previously described $[4,5,11]$. TRAP-positive cells with more than three nuclei were defined as multinucleated osteoclasts. The RAW264 subclones were selected by limiting dilutions. The limiting dilution of the RAW264 cell suspension was seeded into a 96-well plate (1-2 cells/well) and cultured for 10-14 days to form colonies.

\section{Measurement of TRAP activity}

TRAP activity was measured as previously described $[4,12,13]$. Briefly, cells were lysed in extraction buffer $(150 \mathrm{mM} \mathrm{NaCl}, 50 \mathrm{mM}$ Tris, $1 \%$ Nonidet P-40, pH 8.0) supplemented with protease inhibitors (Sigma-Aldrich Japan). A $20 \mu \mathrm{L}$ aliquot of the lysate was then added to $200 \mu \mathrm{L}$ of TRAP buffer $(50 \mathrm{mM} \mathrm{Na}$-acetate, $25 \mathrm{mM} \mathrm{Na}$-tartrate, $0.4 \mathrm{mM}$ $\mathrm{MnCl}_{2}, 0.4 \% \mathrm{~N}, \mathrm{~N}$-dimethylformamide, $0.2 \mathrm{mg} / \mathrm{mL}$ Fast Red Violet, 0.5

${ }^{*}$ Correspondence to: Takuya Notomi, Department of Pharmacology, Osaka Dental University, 8-1 Kuzuhahanazono-cho, Hirakata, Osaka 573-1121, Japan, E-mail: notomi@cc.osaka-dent.ac.jp

Received: October 19, 2019; Accepted: November 04, 2019; Published: November 07, 2019 
$\mathrm{mg} / \mathrm{mL}$ naphthol AS-MX phosphate, $\mathrm{pH}$ 5.0). After incubation for $1 \mathrm{~h}$ (BM) or $2 \mathrm{~h}(\mathrm{RAW})$ at $37^{\circ} \mathrm{C}$, absorbance was measured at $540 \mathrm{~nm}$ using a SpectraMax microplate reader (Molecular Devices, Sunnyvale, CA, USA). Data were normalized to the total protein content, which was determined using a bicinchoninic acid kit (Thermo Fisher Scientific). Each experiment was performed in triplicate.

\section{Gene expression assay}

TRAP activity was measured as previously described [4,5,11]. Total RNA was extracted using an RNeasy kit (Qiagen K. K., Tokyo, Japan) according to the manufacturer's protocol. First-strand cDNA was synthesized from the total RNA using a high-capacity cDNA reverse transcription kit (Thermo Fisher Scientific). The amount of total RNA used in each reaction of synthesis was $1 \mu \mathrm{g}$. Quantitative real-time PCR (qRT-PCR) was performed using a StepOne real-time PCR system (Thermo Fisher Scientific), SYBR Green (Fast SYBR Green master mix, Thermo Fisher Scientific), and specific forward and reverse primers. According to the manufacturer's protocol, the conditions for the reaction were $20 \mu \mathrm{L}$ per well, including $10 \mu \mathrm{L}$ of SYBR Green, $1 \mu \mathrm{L}$ of cDNA template, $0.15 \mu \mathrm{L}$ of $100 \mu \mathrm{M}$ forward and reverse primers, and $8.7 \mu \mathrm{L}$ of RNase-free water. The thermal cycling conditions were as follows: $95^{\circ} \mathrm{C}$ for $20 \mathrm{~s}$, followed by 40 cycles of $95^{\circ} \mathrm{C}$ for $3 \mathrm{~s}$ and $60^{\circ} \mathrm{C}$ for $30 \mathrm{~s}$. Transcript levels were normalized relative to those of glyceraldehyde-3-phosphate dehydrogenase (GAPDH). The quantitative analysis was automatically performed by the StepOne software (Thermo Fisher Scientific). The forward and reverse primer sequences were as follows: TPC2, 5'-GAAGCACAGGACCAGGAGAG3', 5'-AAAGGCCCGGTTCTGAGTAT-3'; GAPDH, 5'-AGAAGGTGGTGAAGCAGGCAT-3' and 5'-CGAAGGTGGAAGAGTGGGAGTTG-3'. The GAPDH signal was identical between the experimental groups.

\section{Statistical analysis}

Values are expressed as the mean \pm standard error of the mean and all values were analyzed using the analysis of variance. The Tukey's honest significant difference test was applied as a post-hoc test. A P-value of $<0.05$ was considered statistically significant. All analyses, including correlation coefficients, were performed using the SPSS software (SPSS Japan, Tokyo, Japan).

\section{Results}

\section{RAW264 subclones exhibit different responses to RANKL stimulation}

To generate the RAW264 subclones, we used the limiting dilution method. As such, we succeeded in establishing 26 subclones for which we then measured individual TRAP activity (Figure 1A). The subclones showed different responses to RANKL stimulation $(50 \mathrm{ng} / \mathrm{mL})$. Clone13 and 15 were sensitive to RANKL stimulation, while Clone10 was insensitive (Figure 1B). To confirm the presence of multinucleated cells, TRAP staining was performed in these three subclones (Figure 2). Large multinucleated cells were observed in both Clone13 and 15, while in Clone10, those cells were hardly observed. At low concentration of RANKL (10 ng/mL), there were no TRAP-positive cells observed in Clone10, while some were present in Clone15. These results indicated that the established subclones had different osteoclastogenesis abilities in response to RANKL stimulation.

Clone13 and 15 formed TRAP-positive, large multinucleated cells at 5 days after the addition of RANKL $(50 \mathrm{ng} / \mathrm{mL})$. Asterisks indicate TRAP-positive, large multinucleated cells. Bar $=100 \mu \mathrm{m}$.
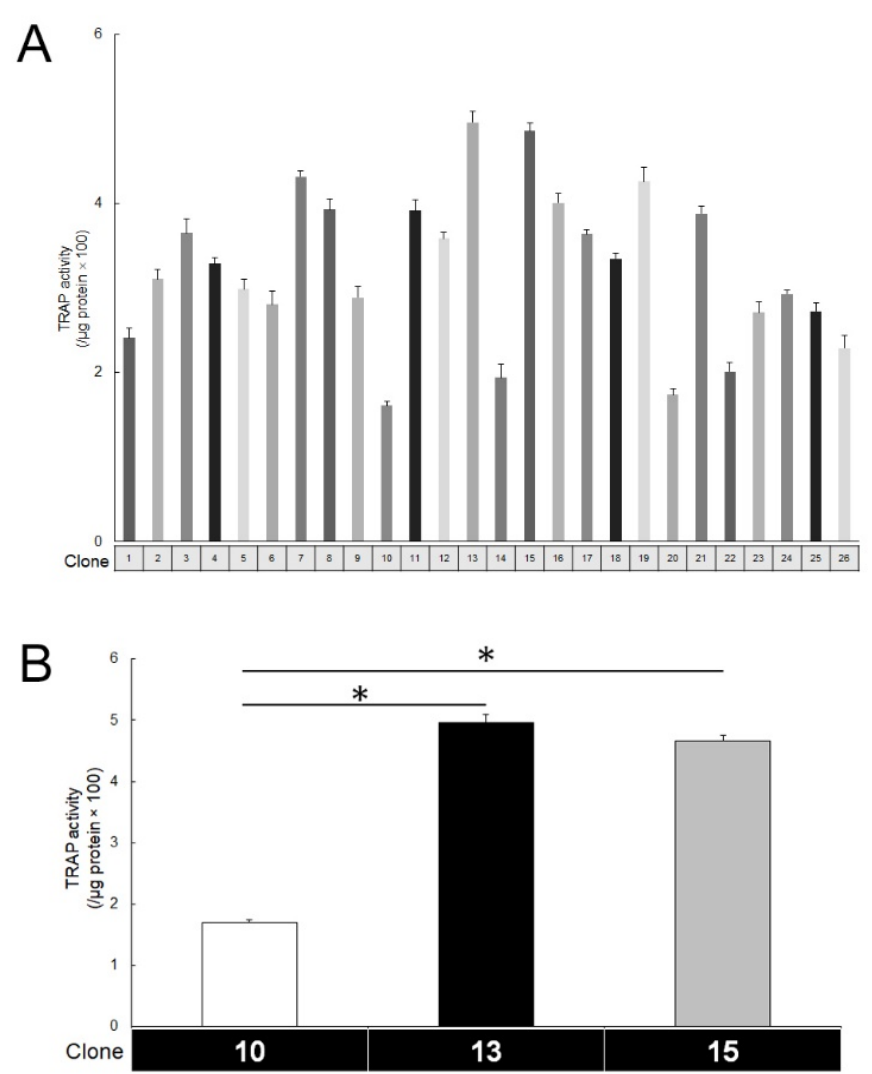

Figure 1. TRAP activity in RAW264 subclones. (A. Each clone exhibited different values of TRAP activity. TRAP activity was measured at 3 days after the addition of RANKL (50 $\mathrm{ng} / \mathrm{mL}$ ). Clone: the number of the subclone. B. Clone13 and 15 were highly sensitive to RANKL stimulation. The addition of RANKL induced high values of TRAP activity in Clone13 and 15 when compared with Clone10. $\left.\left(\mathrm{n}=5,{ }^{*} \mathrm{P}<0.01\right)\right)$

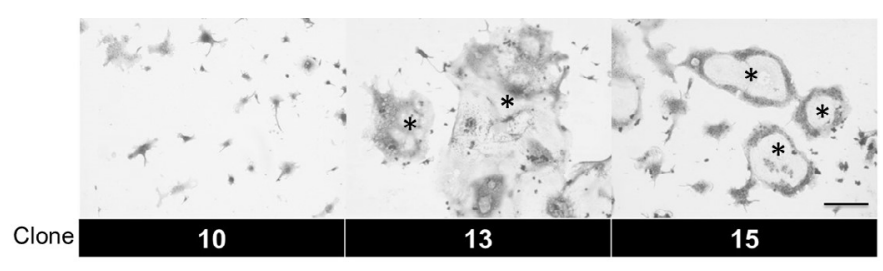

Figure 2. TRAP-positive, large multinucleated cells in RAW264 subclones

\section{TPC2 expression levels are correlated with TRAP activity}

To investigate the relationships between osteoclastogenesis and TPC2 in the established subclones, TPC2 expression levels were examined by qRT-PCR (Figure 3). Clone13 and 15 were found to have high TPC2 expression levels, which were consistent with their high TRAP activity, while Clone10 had low TPC2 expression levels. To further examine the effects of TPC2 on osteoclastogenesis, a correlation analysis was done (Figure 4). There was a positive correlation between TRAP activity and TPC2 expression levels $\left(\mathrm{R}^{2}=0.91, \mathrm{P}<0.01\right)$, suggesting that the differences in sensitivity to RANKL stimulation in the established subclones depend on TPC2 expression levels.

\section{Clone15 has a higher sensitivity to RANKL stimulation than RAW264.7 cells}

RAW264.7 cells, a subclone of RAW264 cells, are widely used to study osteoclast biology as they are more responsive to RANKL stimulation than RAW264 cells [5,9]. As such, to determine whether 
Clone15 has a similar sensitivity to RANKL stimulation, TRAP activity was measured. At 3 days after the addition of RANKL, TRAP activity in Clone10 and 15 was lower and higher than that of RAW264.7 cells, respectively (Figure 5). This suggests that Clone10 and 15 may be useful cell lines for the study of osteoclast biology, similarly to RAW264.7 cells.

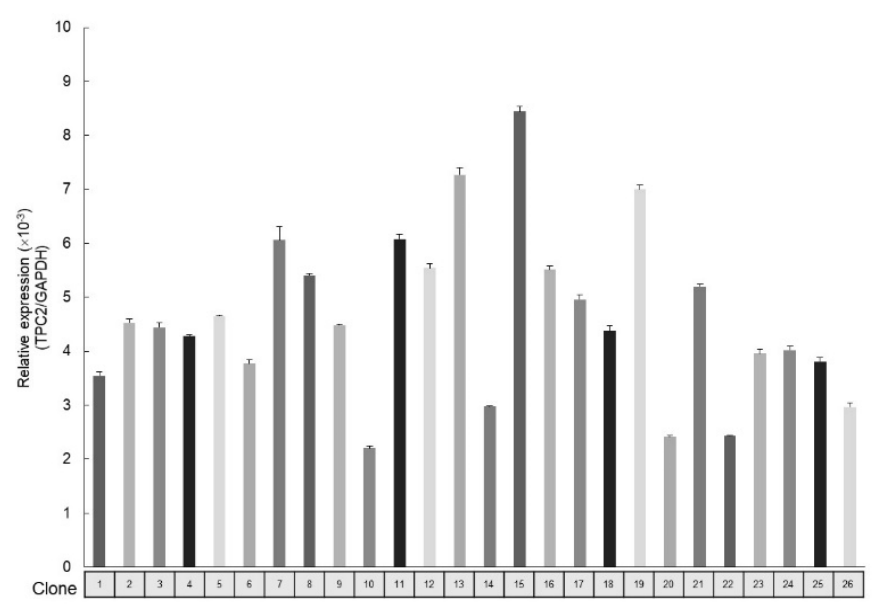

Figure 3. TPC 2 mRNA expression levels in RAW 264 subclones. (The subclones were found to have various TPC2 expression levels. The TPC2 expression level was measured without RANKL stimulation)

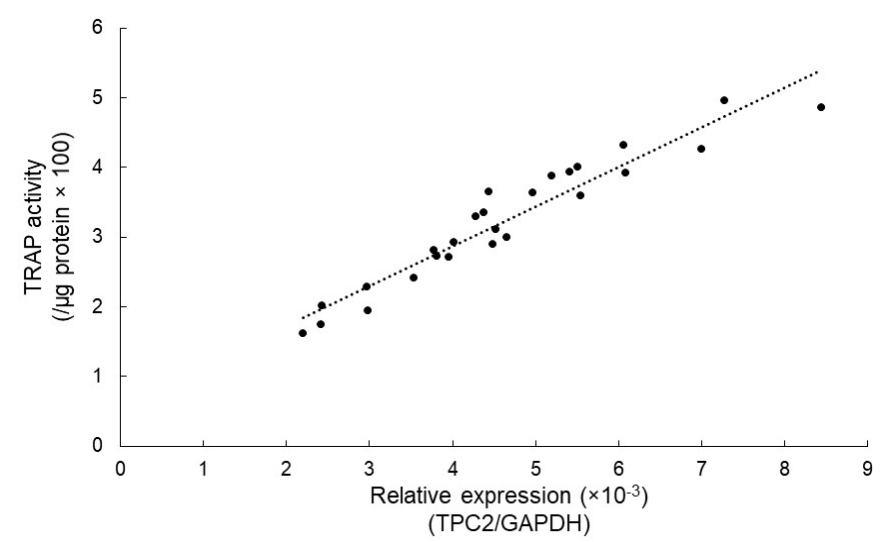

Figure 4. The positive correlation between TRAP activity and TPC2 expression. (The correlation analysis showed a positive correlation between TRAP activity and TPC2 expression in RAW264 subclones)

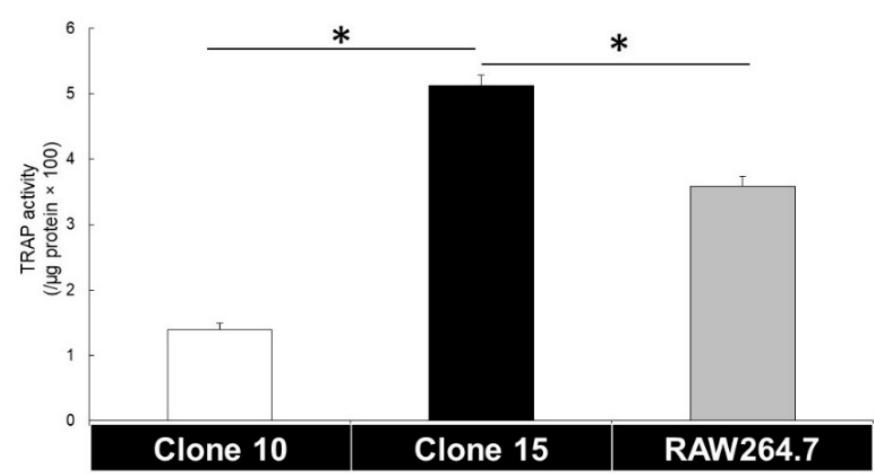

Figure 5. TRAP activity in Clone10, 15, and RAW264.7 cells. (TRAP activity in RAW264.7 cells were in the middle between the three cell lines. $(\mathrm{n}=5 . * \mathrm{P}<0.01))$

\section{Discussion}

The mechanism of osteoclast differentiation and function have been widely investigated based on RANKL/RANK signaling. However, its interaction and relationship with other tissues and cells remain unclear. Although pre-osteoclast-like cells such as RAW264 and RAW264.7 are a useful tool to study osteoclast biology, novel pre-osteoclast cell lines are needed to further our understanding of this process. Here, we established RAW264 subclones with different responses to RANKL stimulation. Clone15 and 10 were found to have high and low sensitivity to RANKL-induced osteoclastogenesis, respectively. Particularly, in Clone15, the addition of RANKL promoted osteoclast differentiation and led to the formation of large multinucleated cells as compared with RAW264.7 cells. Therefore, these novel subclones may be useful models to investigate the molecular mechanisms underlying osteoclast differentiation and function.

TPC2 is a lysosomal $\mathrm{Ca}^{2+}$-permeable channel and TPC2-induced changes in $\left[\mathrm{Ca}^{2+}\right]_{\mathrm{i}}$ promote osteoclast differentiation [4]. Moreover, in our previous study, TPC2 was suggested as a possible target molecule for osteoclastogenesis and osteoporosis treatment [4]. The findings of this current study strongly support this suggestion. The correlation analysis showed a strong relationship between osteoclastogenesis and TPC2 expression. As the RNA samples were extracted before the addition of RANKL, it suggests that TPC2 expression levels in pre-osteoclast are important for osteoclast differentiation. The effects of $\left[\mathrm{Ca}^{2+}\right]_{\mathrm{i}}$ changes in the late stages of osteoclastogenesis are relatively minimal [14]. This suggests that TPC2 levels might not be important in the late stages of osteoclast differentiation.

A limitation of our study is that we only analyzed TPC2 expression levels in the newly generated RAW264 subclones. The molecular mechanisms for osteoclast differentiation in RAW264.7 cells are well understood $[9,13,15]$. Moreover, both RAW264 and 264.7 cells are widely used and the possible genes involved in osteoclastogenesis were identified in these cells. As such, our novel subclones require further analysis to improve their reliability as pre-osteoclast-like cells.

\section{Conclusion}

In summary, we established novel RAW264 subclones which exhibited different responses to RANKL stimulation. Osteoclastogenesis in each subclone was strongly correlated with TPC2 mRNA expression levels. In comparison with RAW264.7 cells, Clone15 and 10 were shown to have higher and lower sensitivity to RANKL-induced osteoclastogenesis, respectively. These results suggest that Clone15 and 10 may be useful models for the study of osteoclast differentiation and function.

\section{Conflicts of interest}

The authors declare that they have no conflicts of interest with the content of this article.

\section{References}

1. Boyle WJ, Simonet WS, Lacey DL (2003) Osteoclast differentiation and activation. Nature 423: 337-342. [Crossref]

2. Karsenty G and Wagner EF (2002) Reaching a genetic and molecular understanding of skeletal development. Dev Cell 2: 389-406.

3. Takayanagi H, Kim S, Koga T, Nishina H, Isshiki M, et al. (2002) Induction and activation of the transcription factor NFATc1 (NFAT2) integrate RANKL signaling in terminal differentiation of osteoclasts. Dev Cell 3: 889-901. 
4. Notomi T, Ezura Y, Noda M (2012) Identification of two-pore channel 2 as a novel regulator of osteoclastogenesis. J Biol Chem 287: 35057-35064. [Crossref]

5. Notomi T, Kuno M, Hiyama A, Nozaki T, Ohura K, et al. (2017) Role of lysosomal channel protein TPC2 in osteoclast differentiation and bone remodeling under normal and low-magnesium conditions. J Biol Chem 292: 20998-21010. [Crossref]

6. Calcraft PJ, Ruas M, Pan Z, Cheng X, Arredouani A, et al. (2009) NAADP mobilizes calcium from acidic organelles through two-pore channels. Nature 459: 596-600.

7. Galione A, Morgan AJ, Arredouani A, Davis LC, Rietdorf K, et al. (2010) NAADP as an intracellular messenger regulating lysosomal calcium-release channels. Biochem Soc Trans 38: 1424-1431.

8. Watanabe T, Kukita T, Kukita A, Wada N, Toh K, et al. (2004) Direct stimulation of osteoclastogenesis by MIP-1alpha: evidence obtained from studies using RAW264 cell clone highly responsive to RANKL. J Endocrinol 180: 193-201.

9. Cuetara BL, Crotti TN, O'Donoghue AJ, McHugh KP (2006) Cloning and characterization of osteoclast precursors from the RAW264.7 cell line. In Vitro Cell Dev Biol Anim 42: 182-188. [Crossref]

10. Sakai H, Moriura Y, Notomi T, Kawawaki J, Ohnishi K, et al. (2010) Phospholipase $\mathrm{C}$-dependent $\mathrm{Ca} 2+$-sensing pathways leading to endocytosis and inhibition of the plasma membrane vacuolar H+-ATPase in osteoclasts. Am J Physiol Cell Physiol 299 : C570-C578.
11. Notomi T, Kuno M, Hiyama A, Ohura K, Noda M, et al. (2015) Zinc-induced effects on osteoclastogenesis involves activation of hyperpolarization-activated cyclic nucleotide modulated channels via changes in membrane potential. J Bone Miner Res 30: 16181626.

12. Notomi T, Kuno M, Hiyama A, Ezura Y, Honma M, et al. (2015) Membrane depolarization regulates intracellular RANKL transport in non-excitable osteoblasts. Bone 81: 306-314.

13. Notomi T, Kuno M, Hiyama A, Ohura K, Noda M, Et al. (2015) Zinc-Induced Effects on Osteoclastogenesis Involves Activation of Hyperpolarization-Activated Cyclic Nucleotide Modulated Channels via Changes in Membrane Potential. J Bone Miner Res 30: 1618-1626.

14. Masuyama R, Vriens J, Voets T, Karashima Y, Owsianik G, et al. (2008) TRPV4 mediated calcium influx regulates terminal differentiation of osteoclasts. Cell Metab 8: $257-265$

15. Mori H, Sakai H, Morihata H, Kawawaki J, Amano H, et al. (2003) Regulatory mechanisms and physiological relevance of a voltage-gated $\mathrm{H}+$ channel in murine osteoclasts: phorbol myristate acetate induces cell acidosis and the channel activation. J Bone Miner Res 18: 2069-2076.

Copyright: $(02019$ Notomi T. This is an open-access article distributed under the terms of the Creative Commons Attribution License, which permits unrestricted use, distribution, and reproduction in any medium, provided the original author and source are credited. 